\title{
Grosor de Íntima-Media Carotídea en Niños Sanos por Técnica Ecográfica Computarizada
}

\author{
ANIBAL ESPINOZA G. ${ }^{1,2,3}$, CATALINA BAGÉS M. ${ }^{4}$, \\ CATALINA LE ROY O. ${ }^{1}$, CARLOS CASTILLO D. ${ }^{1}$ \\ 1. Departamento de Pediatría, Facultad de Medicina Campus Centro, Universidad de Chile. \\ 2. Clínica Alemana de Santiago. \\ 3. Servicio de Radiología, Complejo Hospitalario San Borja-Arriarán. \\ 4. Instituto de Nutrición y Tecnología de los Alimentos (INTA), Universidad de Chile.
}

\begin{abstract}
Arterial intima-media thickness in healthy Chilean children using an ultrasonographic computerized technique
\end{abstract}

Introduction: Vascular endothelium is a target organ in metabolic syndrome. Ultrasound measurement of intimae-media thickness (IMT) is used in adults to predict cardiovascular risk, but normal values in children are unknown. Objective: To describe arterial intima-media thickness in healthy children, by means of an ultrasonographic technique and a specific software. Subjects and Methods: Seventy-four healthy children, 39 males, ages 5-15 y.o., body mass index (BMI) 10-85 percentile were selected from an upper-middle socioeconomic background. Subjects were grouped by age. Three measurements were carried out to each subject at the middle third of the right common carotid for maximal intima-media thickness using a Phillips iU22 ultrasound system; a 12-5 MHz lineal transducer and QLAB advanced quantification software, which includes a specific program for automated IMT measurements, were used. Weight, stature, BMI, and abdominal circumference were registered. Results: No significant differences in the IMT values according to age or gender were found. Median IMT was $0.41 \mathrm{~mm}(0.40-0.56 \mathrm{~mm}) ; 5$ children presented IMT $0.50-0.56 \mathrm{~mm}$ ( $>75$ th percentile). The median IMT for those children with abdominal circumference $<50$ th percentile was the same for those over 50th percentile $(0.41 \mathrm{~mm})$. Conclusions: Measurements of carotid intima-media thickness for healthy Chilean children were found to be in the expected range, in relation with adult known values.

(Key words: Arterial intima-media, thickness, ultrasonography, healthy children).

Rev Chil Pediatr 2011; 82 (1): 29-34

\section{RESUMEN}

Introducción: Uno de los sitios de daño asociado a síndrome metabólico es el endotelio vascular; la medición ecográfica del grosor de íntima-media carotídea (GIMC) es usado en adultos como un indicador de daño vascular, pero no se conocen valores de normalidad en niños. Objetivo: Estudiar el grosor de íntima-media

Trabajo recibido el 01 de septiembre de 2010, devuelto para corregir el 28 de septiembre de 2010, segunda versión el 17 de diciembre de 2010, tercera versión el 06 de enero de 2011, aceptado para publicación el 31 de enero de 2011.

Correspondencia a:

Dr. Aníbal Espinoza G.

E-mail: anibalespinoza2005@yahoo.com 
carotídea mediante programa computacional específico, en niños chilenos sanos. Pacientes y Método: Se seleccionaron 72 niños (percentiles 10-85 de IMC sin enfermedad conocida), 5 a 15 años de edad, de estratos socioeconómicos medio-altos, 33 niñas y 39 varones, agrupados por tramos de edad; se les efectuó y promedió 3 mediciones ecográficas en tercio medio de arteria carótida común derecha, mediante equipo Phillips modelo iU22; se usó un transductor lineal 12-5 MHz y un software de cuantificación avanzada QLAB, con programa específico de medición automática para grosor de GIMC. Además se les midió: peso, talla, IMC, perímetro abdominal (PA). Se solicitó previamente el consentimiento escrito a padres y niños. Resultados: No hubo diferencias en las mediciones de GIMC de acuerdo a edad o sexo, la mediana fue de 0,41 mm (0,40-0,56 mm); hubo 5 niños entre 0,50 y $0,56 \mathrm{~mm}$ (> percentil 75); no hubo correlación con PA o IMC; los niños con PA < percentil 50 tuvieron una mediana de GIMC igual a aquellos con PA $>$ percentil $50(0,41 \mathrm{~mm})$. Conclusiones: Las mediciones de grosor de íntima-media carotídea de niños y adolescentes chilenos sanos mediante técnica ecográfica y programa computarizado, están dentro de valores esperados para la edad, comparados con valores conocidos de adultos.

(Palabras clave: Intima media arterial, grosor, ecografia, niños sanos).

Rev Chil Pediatr 2011; 82 (1): 29-34

\section{Introducción}

Uno de los sitios de daño asociado a síndrome metabólico es el endotelio vascular; la medición ecográfica del grosor de íntima-media arterial (GIMA) es usado en adultos como un indicador de daño vascular, dada su reconocida asociación con enfermedad cardiovascular.

Desde los estudios realizados en la década de los de 90 en Bogalusa ${ }^{1}$ hay evidencia que el daño vascular puede comenzar a desarrollarse desde los primeros años de vida y en sujetos aparentemente sanos.

El incremento anormal del grosor de IM de carótida común, se relaciona con múltiples factores de riesgo cardiovascular como son: sexo masculino, diabetes, colesterol total y tabaquismo en adultos ${ }^{2}$. Hay escasos estudios a nivel internacional analizando el GIMA en niños y adolescentes $\operatorname{sanos}^{3}$; y en especial se conocen escasos datos de valores de normalidad en niños chilenos ${ }^{4}$.

El advenimiento de tecnologías automáticas para la medición ecográfica del GIMA, que se desarrollan desde fines de los años 90, hoy son una realidad como software en los equipos ecográficos modernos, ello ha simplificado la obtención de varias mediciones de GIMA en forma automática, rápida y con menor sesgo operador dependiente ${ }^{5}$.

Nuestro objetivo fue estudiar mediante un programa computacional ecográfico específi- co, las variaciones de las mediciones de grosor de íntima-media carotídea (GIMC) en niños chilenos aparentemente sanos.

\section{Pacientes y Método}

En una muestra por conveniencia, se seleccionaron 72 niños chilenos, 39 varones, eutróficos (IMC percentiles 10-85 de los estándares en uso en Chile del CDC según edad y sexo), edad entre 5 y 15 años, sin antecedentes mórbidos conocidos ni de enfermedad reciente, sin uso de medicamentos ni hábito tabáquico, $\mathrm{y}$ asistentes a colegios privados que atienden estratos socioeconómicos medio-altos.

Inmediatamente después de las mediciones antropométricas, se efectuaron las mediciones ecográficas, con el niño tranquilo, en decúbito supino y el cuello extendido y rotado a la izquierda. Con equipo Phillips modelo iU22, se les efectuó y promedió 3 mediciones estandarizadas en arteria carótida común derecha (CCD) a 1-2 cm bajo la bifurcación con transductor lineal de alta frecuencia (12 -5 MHz) en modo B, en la pared lejana al transductor ${ }^{6}$. La medición se realizó con software de cuantificación avanzada QLAB, con un programa específico de medición automática para GIMC. En un formato de video digital, se adquieren 300 imágenes; entre éstas, se seleccionaron las de mejor calidad para la visualización de la pared 
arterial distal y dentro de ellas se eligió el área de la pared arterial a medir. Con la imagen y área a medir elegida, la medición se realizó en forma automática. El software calculó un promedio del espesor en un segmento de $1 \mathrm{~cm}$ de longitud; el programa también entrega el porcentaje de éxito de la medición automática del segmento elegido manualmente, es decir evalúa la calidad de la imagen para ser susceptible de medir automáticamente (se recomienda un éxito $\geq$ a $85 \%$, en este trabajo usamos un éxito $\geq 95 \%$ ). Con este software el límite inferior de detección de GIMA es de 0,40 mm. Todas las mediciones fueron realizadas por un solo radiólogo con entrenamiento previo en esta técnica. Además se les midió: peso, talla, IMC y perímetro abdominal. No se consideró la toma de muestras de sangre para variables metabólicas. El protocolo fue autorizado previamente por el Comité de Investigaciones y Ética del Departamento de Pediatría Facultad de Medicina Campus Centro y por el Comité de Ética de Clínica Alemana. Se solicitó el consenti- miento escrito a padres y el asentimiento a los niños. Las datos se expresaron como mediana y dispersión percentilar.

\section{Resultados}

Las características de los niños estudiados se muestran en la tabla 1. No hubo diferencias en IMC de acuerdo a sexo (varones: 17,9 \pm 1,9 ; niñas: $18,0 \pm 2,2 \mathrm{~kg} / \mathrm{m}^{2}$ ).

El coeficiente de variación intraindividual de las tres mediciones para cada niño fue de un $2,4 \%$. El tiempo de ejecución fue de 5-10 minutos (rango estimado).

Las mediciones de GIMC variaron entre 0,40 y $0,56 \mathrm{~mm}$ con una mediana de $0,41 \mathrm{~mm}$; no hubo diferencias de acuerdo a edad o sexo. Cinco niños presentaron valores sobre el percentil 75 de este grupo (>0,50 mm) (figura 1). No hubo correlación con perímetro abdominal o IMC; la mediana de GIMC para los niños < percentil 50 de PA fue igual a la de aquellos sobre el percentil 50 de PA $(0,41 \mathrm{~mm})$.
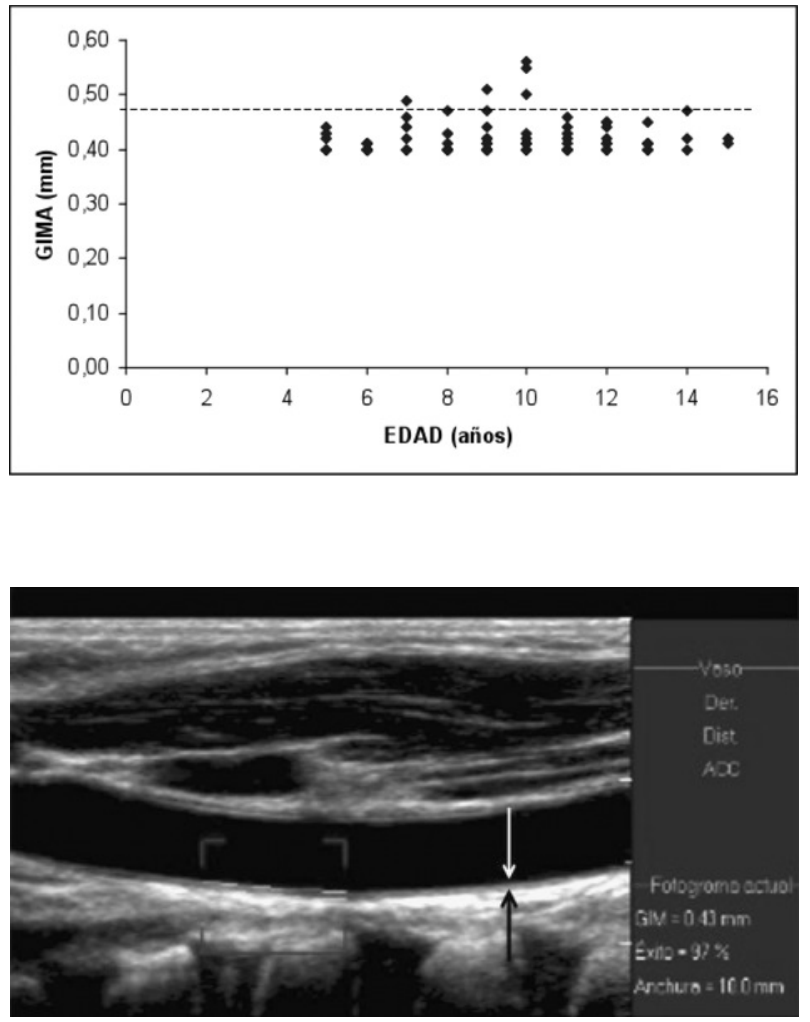

Figura 1. Valores de grosor de íntima media de arteria carótida derecha (GIMA) según edad, en escolares y adolescentes chilenos aparentemente sanos. La línea punteada expresa el percentil 75 de los datos analizados $(0,49 \mathrm{~mm})$.

Figura 2. Ecografía de íntima media carotídea de uno de los escolares estudiados. Las flechas muestran el grosor de íntima media. A izquierda se observa un recuadro de 10,0 $\mathrm{mm}$ de extensión donde se realiza la medición automática de GIMA, a derecha se lee el valor promedio obtenido y el \% de éxito de la medición computacional. 
Tabla 1. Características de los escolares y adolescentes aparentemente sanos estudiados $(n=72)$

\begin{tabular}{|lc|}
\hline Variable & $\mathbf{x} \pm \mathbf{D E}$ \\
\hline Edad (años) & $9,5 \pm 2,7$ \\
\hline Género (M/F) & $39 / 33$ \\
Peso $(\mathrm{kg})$ & $34,8 \pm 10,3$ \\
\hline IMC $\left(\mathrm{kg} / \mathrm{m}^{2}\right)$ & $18,0 \pm 2,1$ \\
\hline Puntaje z Talla/Edad & $-0,1 \pm 0,93$ \\
\hline
\end{tabular}

\section{Discusión}

Las mediciones de grosor íntima-media de arteria carótida derecha encontradas en estos escolares y adolescentes aparentemente sanos tienen una variabilidad concordante con las observadas en otros estudios semejantes en adolescentes o adultos. La falta de progresión del GIMC dentro del rango de edades estudiado, puede tener relación con la normalidad esperada para estas edades, con un aumento de ese grosor en edades posteriores en la vida adulta $^{7}$.

El sistema automático para la medición de GIMA está proporcionando una mayor confiabilidad comparado con las mediciones manuales. Está basado en un software computacional que mide el grosor máximo de la pared arterial dentro de un área predeterminada por el operador. Nuestras mediciones fueron hechas por un solo operador con un solo equipo ecográfico de última generación, usando el método automático (equipo Phillips IU22 con software de cuantificación avanzada QLAB) (figura 2).

Las medidas se realizaron en un tiempo significativamente menor a una medición manual. En estudios previos en adultos, con el programa automático se demoraban entre la mitad y un tercio del tiempo en que se lograban con la medición manual ${ }^{5}$. La medición automática del GIMA, realizada por el software sobre una imagen digital de la pared arterial se basa en tres principios; diferente intensidad ecogénica, gradiente ecogénica y continuidad de bordes ${ }^{5}$. La resolución espacial de los equipos en uso para medir GIMA en forma digital es de 0,4 $\mathrm{mm}^{3}$. Dado que la medición que se está buscando es engrosamiento de la pared arterial no se justifica que el software mida valores menores que los señalados, incluso en niños.

Wendelhag et $\mathrm{al}^{5}$, habían demostrado variabilidad entre operadores, cuando se comparaban un operador sin experiencia previa y otro con experiencia con uso de la técnica manual de medición de GIMA. Las diferencias observadas son significativas. Cuando las medidas son realizadas con la técnica automática no existen diferencias o son menores.

Las comparaciones hechas del grosor arterial en cadáveres, medidos con técnicas histológicas y mediciones ecográficas muestran una subestimación de los valores ecográficos hasta en un $20 \%{ }^{8}$. Los estudios de las mediciones en diferentes ubicaciones en la arteria, revelan que al medir GIMA en la pared arterial lejana al transductor, la subestimación antes descrita se reduce a valores no significativos ${ }^{8}$. Actualmente los programas computacionales obtienen las medidas de GIMA en la pared lejana. Todas nuestras medidas fueron hechas en la pared lejana de la arteria carótida común derecha.

Diferentes arterias de la economía son susceptibles de ser evaluadas con medición de IM; condiciones favorables son su cercanía con la superficie cutánea y un trayecto paralelo a ésta. La gran mayoría de las publicaciones comunican mediciones en la ACCD. Otras arterias posibles de medir son carótida interna, carótida externa, bulbo carotídeo, ilíaca común y femoral. La arteria carótida común tiene ventajas comparativas: es la arteria más asequible, más paralela y cercana a la piel, posible de ser evaluada en casi el $100 \%$ de los pacientes y presenta la menor dispersión de datos inter e intra operador ${ }^{2}$. Las mediciones de la ACC derecha no difieren del lado contralateral ${ }^{9}$, excepto el trabajo de González et $a^{10}{ }^{10}$, donde las medidas de GIMA de carótida derecha miden 2,2 a 3,1\% más que la izquierda. Mediciones de GIMC en el bulbo carotídeo son técnicamente más difíciles de realizar ${ }^{2}$. La arteria aorta es la localización elegida para medir GIMA en recién nacidos, No es susceptible de medir en niños, por interferencia del gas intestinal.

En un metanálisis de 23 estudios de la literatura de lengua inglesa hasta el año 1997, el valor medio en la población (median popula- 
tion values) sin detallar la etnia de los pacientes estudiados ${ }^{9}$ dio valores entre 0,4 y $1,0 \mathrm{~mm}$, con un incremento de 0,01 a $0,03 \mathrm{~mm}$ por año en edades adultas. Un estudio más reciente ${ }^{11}$ también en adultos sanos, mostraba una progresión pequeña de los promedios de GIMA desde $0,44 \mathrm{~mm}$ en $<30$ años a 0,54 en $>50$ años; el percentil 75 en el mismo estudio aumentaba desde 0,48 a 0,62. Estos datos son plenamente concordantes con los nuestros.

Jourdan et al el año $2005^{7}$, presentaban una serie de 247 adolescentes aparentemente sanos de entre 10 y 20 años. Los valores de GIMC no diferían significativamente entre hombres y mujeres. El GIMC presentaba un leve incremento asociado con la edad y una mejor asociación positiva con la talla. Las mediciones de GIMC fueron hechas manualmente, con medidas mínimas y máximas de 0,374 y $0,404 \mathrm{~mm}$, respectivamente. Estos valores son inferiores a los nuestros, fenómeno que puede estar en relación al uso de técnica manual de medición. Este fenómeno también se repite en un grupo control de 20 niños chilenos en el trabajo de Delucchi et $\mathrm{al}^{4}$, con valores ligeramente inferiores a los nuestros.

Los grosores de GIMC realizadas con el sistema computacional son comparativamente más bajos que los observados por Barja et $\mathrm{al}^{12}$, tomando los resultados en ese estudio del grupo de niños y adolescentes con cero factores de riesgo de síndrome metabólico $(0,41$ vs 0,49 $\mathrm{mm})$. En este trabajo de Barja et al ${ }^{12}$, los niños del grupo con 0 agregación de componentes de SM, tenían edades entre 9,4 y 13,4 años, estos niños parecen no ser totalmente homologables a niños sanos a pesar de no tener ningún componente de SM; la media de IMC era de 18,7 $\pm 2,7$, valores que según los estándares de la OMS 2007 incluyen a niños en condición de sobrepeso, e incluso de obesidad.

Nuestra muestra de niños fue de edades algo menores a los diferentes estudios en niños sanos descritos en la literatura, con una media de 9,6 años y con límites entre 5 y 15 años. En varios estudios las edades menores eran de 11 años ${ }^{3}$.

Un trabajo reciente en niños chilenos de Balboa et $\mathrm{al}^{13}$, mostraba valores promedios de GIMC de 0,26 mm con una DE de 0,04 en niños $>11$ años, pero con mediciones manua- les de GIMC. Las mediciones con software muestran valores consistentemente más altos que las mediciones manuales, discriminando valores sobre $0,40 \mathrm{~mm}^{13}$, al ser ésta una resultante de la resolución espacial de los equipos ecográficos.

El método computacional de evaluación del grosor IM en carótida, está avalado en la literatura; su gran ventaja es su reproducibilidad con baja variabilidad intra e interoperador ${ }^{5}$.

¿Cuál es el significado del engrosamiento de IM?, es una pregunta aun no resuelta totalmente; hay un incremento normal del grosor de la pared arterial con la edad, que puede considerarse como un fenómeno no asociado a la aparición del fenómeno ateroesclerótico. Dentro de este proceso fisiopatológico, la aparición de estrías grasas y placas de ateromas es focal y no difuso, como lo es el engrosamiento de GIMA. Otra posibilidad es que el aumento del GIMA sea como respuesta a la hipertensión arterial, representando una hipertrofia o hiperplasia vascular reactiva a la sobrecarga; este proceso implicaría que el engrosamiento estaría partiendo con la aparición de la hipertensión arterial, más propio del adolescente obeso y con síndrome metabólico.

El estudio de González et $\mathrm{al}^{10}$, en adultos jóvenes sanos mostraba que GIMC se asociaba a IMC y presión arterial, con medias en torno a $0,7 \mathrm{~mm}$. En general valores próximos o mayores a $1 \mathrm{~mm}$ de grosor IM en adultos se asocian a riesgo cardiovascular y cerebrovascular ${ }^{11}$.

En resumen, nuestro estudio en escolares y adolescentes chilenos aparentemente sanos, muestra un grosor de íntima media carotídea con una mediana de $0,41 \mathrm{~mm}$, mediante mediciones ecográficas automáticas con un programa específico. Un sub-grupo limitado de estos presenta valores aumentados sobre $0,49 \mathrm{~mm}$, requiriéndose en estudios futuros su evaluación metabólica y un seguimiento a largo plazo.

\section{Agradecimientos}

Agradecemos sinceramente la colaboración del personal de secretaría y del Servicio de Radiología de Clínica Alemana para llevar a cabo las mediciones. 


\section{Referencias}

1.- Berenson GS, Srinivasan SR, Bao W, Newman WP 3rd, Tracy RE, Wattigney WA: Association between multiple cardiovascular risk factors and atherosclerosis in children and young adults. The Bogalusa Heart Study. N Engl J Med 1998; 338: 1650-6.

2.- Roman MJ, Naqvi TZ, Gardin JM, Gerhard-Herman M, Jaff M, Mohler E: American Society of Echocardiography report. Clinical application of non-invasive vascular ultrasound in cardiovascular risk stratification: a report from the American Society of Echocardiography and the Society for Vascular Medicine and Biology. Vasc Med 2006; 11: 201-11.

3.- Urbina E, Williams R, Alpert B, et al: Noninvasive assessment of subclinical atherosclerosis in children and adolescents. Hypertension 2009; 54: 919-50.

4.- Delucchi A, Dinamarca H, Gainza H, Whitttle C, Torrealba I, Iñiguez $G$ : Carotid intima-media thickness as a cardiovascular risk marker in pediatric end-stage renal disease patients on dialysis and in renal transplantation. Transplant Proc 2008; 40: 3244-6.

5.- Wendelhag I, Liang Q, Gustavsson T, Wikstrand J: A new automated computerized analyzing system simplifies readings and reduces the variability in ultrasound measurement of intima-media thickness. Stroke 1997; 28: 2195-200.

6.- Litwin $M$, Niemirska A: Intima-media thickness measurements in children with cardiovascular risk factors
(Review). Pediatr Nephrol 2009; 24: 707-19.

7.- Jourdan $C, W u$ E, Litwin $M$, et al: Normative values for intima-media thickness and distensibility of large arteries in healthy adolescents. J Hypertens 2005; 23: 1707-15.

8.- Wong M, Edelstein J, Wollman JM, Bond G: Ultrasonicpathological comparison of the human arterial wall. Verification of intima-media thickness. Arterioscl Thromb 1993; 13: 482-6.

9.- Kanters SDJM, Algra A, van Leeuwen MS, Banga $J D$ : Reproducibility of in vivo carotid intima-media thickness measurements: A review. Stroke 1997; 28: 665-71.

10.- González J, Wood J, Dorey F, Wren T, Gilsanz V: Reproducibility of carotid intima-media thickness measurements in young adults. Radiology 2008; 247: 465-71.

11.- Simon A, Gariepy J, Chironi G, Megnien JL, Levenson $J$ : Intima-media thickness: a new tool for diagnosis and treatment of cardiovascular risk. J Hipertens 2002; 20: 159-69.

12.- Barja S, Acevedo M, Arnaiz P, et al: Marcadores de aterosclerosis temprana y síndrome metabólico en niños. Rev Med Chile 2009; 137: 522-30.

13.- Balboa P, Torrejón C, Hevia M, Ureta E, Valenzuela X: Grosor de la íntima-media y rigidez de la arteria carótida en adolescentes obesos y su relación con el síndrome metabólico. XLVII Reunión Sociedad Latinoamérica de Investigación Pediátrica (SLAIP), Asunción, Paraguay Octubre 2009 (Resumen 36). 